\title{
Deutsche Dermatologische Gesellschaft
}

Am 26. III. 1918 fand in Berlin eine außerordentlîche Sítzung des Ausschusses statt. In dieser Wurden folgende, für die Mitglieder der Gesellschaft wichtige Beschlüsse gefaßt:

Es wird besehlossen, von der Wahl eines Generajsekretärs für jetz · abzvsehen und die Neubesfcallung des Vorstandes statutengemäß bis zur Wiedererneuerung des Ausschusses bei der allgemeinen nächsten Versammlung zu verschieben.

Im Ans $\beta$ hluß an den Kassenbericb.t wird besohlossen, während: des Krieges aueh weiterhin die Mitgliederbeiträge nicht einzuziehen. .,

Von einer allgemeinen Versammlung während des Krieges soil zunäehst jedenfalls abgesehen Werden.

Die Bestrebungen der deutsehen Universitätslehrer, bei den zu-ständigen Behörden des Reiebes und der Einzelstaaten voile Prüíung in Dermatologie, Ausbau der dermatologischen Kliniken usw. zu erreichen^ sollen von der D. D. G. energiseh unterstützt werden.

Personalien.

Der a.-o. Professor Dr. F. Lewandowsky in Basel, Direktor der Dermatologischen Universitätsklinik, wurde zum ordentlichen Professor er-nannt.

An der nouon Universität in Preßburg ist Priv.-Dozent Dr. Franz Vereß (Klausonburg) zum o. ö. Professor der Derrratologie ernannt worden.

Geh. Med.-Rat Prof. Dr. E. Lesser, Direktor der Universitätsklinik für Hautkrankheiten in Berlin, ist im Alter von 66 Jahren am 5. Juni ge-storben. Nachruf folgt.

Tagesnachrichten.

Am 28. IX. wird unter dem Vorsitz von Geh.-Rat Prof. Dr. Herxheimer in Frankfurt a. M. eine Dermatologentagung der südwestdeutschen Derma-tologen-Vereinigung stattfinden, zu der au $\beta \mathrm{h}$ die Mitglieder der nieder-rheinis $\beta$ h-westfälisehen Dermatologen-Vereinigung und der Berliner $\mathrm{i}^{1} / 8 \mathrm{~d}$ Wiener Dermatologischen Gesellschaft geladen werden sollen Unser hoch-ges $\not$ hätzter Mitherausgeber darf des Dankes aller Kollegen dafür sicher sein, daß er trotz des Krieges uns wieder Gelegenheit gibt, unsere Erfahrungen ausz, itausohen. Wie wir hören, werden au $\beta h$ hochwichtige und neue Tat-saehen auf dem Gebiet der Syphilisbehandlung von Ehrlichs Naehfoger Geh.-Rat Prof. KolL·, mitgeteilt Werden. 